UNIVERSITY of PENNSYLVANIA

Department of Physics

David Rittenhouse Laboratory

Philadelphia PA 19104-6396

PREPRINT UPR-0127MT

July 1993

\title{
The Skyrme model description of the spin-orbit force
}

R. D. Amado, Bin Shao and Niels R. Walet

Submitted to Physics Letters B 


\title{
The Skyrme model description of the spin-orbit force
}

\author{
R. D. Amado, Bin Shao and Niels R. Walet* \\ Department of Physics, University of Pennsylvania, \\ Philadelphia, PA 19104
}

September 14, 2018

\begin{abstract}
We examine the derivation of the spin-orbit force from the Skyrme model. We find substantial agreement with phenomenological potentials. We show that a systematic and simultaneous treatment of all components of the kinetic energy introduces a minus sign relative to previous calculations for the iso-spin independent part of the interaction. We also explain the smallness of the iso-spin dependent part of the spin-orbit potential.
\end{abstract}

The challenge of obtaining the $N N$ interaction from a QCD inspired starting point has recently been given new impetus by results in the Skyrme model [1], 2]. Careful treatment of the non-linear nature of the model and of quantum corrections leads to significant central attraction, in substantial agreement with phenomenology. There is even a strong short range repulsion, though its detailed description certainly lies outside the scope of the Skyrme model with pions only. Combined with the well known Skyrme treatment of one pion exchange and the tensor force, all this yields a satisfactory picture of the static part of the $N N$ interaction that gives a good description of the phase shifts. The next step is to consider the non-static parts of the interaction. Here we consider the spin-orbit force. The dominant part of the phenomenological spin-orbit interaction is iso-spin independent. Attempts to reproduce it from the Skyrme model have given the correct order of magnitude, but the wrong sign [3, 国. In this note we point out that a careful treatment of the conversion from velocities to canonical momenta in fact reverses that sign and hence yields a spin-orbit interaction in substantial agreement with the data. We also show that a small iso-spin dependent spin-orbit force emerges naturally.

The principal ingredients in the successful static $N N$ interaction calculations are numerically "exact" solutions for the baryon number two $(B=2)$ system at finite

*address after Sept. 1, 1993: Institüt für theoretische Physik III, Universität Erlangen-Nürnberg, D-91058 Erlangen, Germany 
separation, and quantum corrections implemented through the Born-Oppenheimer approximation. The exact results differ considerably from earlier ones based on the simple product Ansatz largely because the static interaction energy arises as the relatively small difference of two large numbers. Cancellations must therefore be treated with care. Although a full treatment of the non-static terms should also be done exactly, there are no corresponding cancellations in this case, and one can hope to get insight from the product Ansatz. It is for that reason that the wrong sign for the spin-orbit interaction obtained in earlier product Ansatz calculations was particularly disappointing [3, 4.

Since there has not been a complete study of the kinetic energy of the Skyrme model, even though pieces can be found in the literature [3, 4, 5], we decided to undertake such a study. A part of our purpose was to understand the puzzle of the spin-orbit sign. In this note we sketch the steps in our evaluation, and show that there is a simple solution to the sign problem.

The Skyrme model [6, 7] is a non-linear field theory that can be realized in terms of an $\mathrm{SU}(2)$-valued matrix field $U$, with Lagrangian density

$$
\mathcal{L}=\frac{f_{\pi}^{2}}{4} \operatorname{Tr}\left[\partial_{\mu} U(x) \partial^{\mu} U^{\dagger}(x)\right]+\frac{1}{32 g^{2}} \operatorname{Tr}\left[U^{\dagger} \partial_{\mu} U, U^{\dagger} \partial_{\nu} U\right]^{2}+\frac{f_{\pi}^{2} m_{\pi}^{2}}{4}\left[\operatorname{Tr}\left(U+U^{\dagger}\right)-4\right] .
$$

The model is covariant, as well as invariant under global SU(2)-rotations that are identified with the iso-spin symmetry. The only parameters in the model are the pion mass, $m_{\pi}$, the pion decay constant, $f_{\pi}$, and the dimensionless parameter, $g$. It is customary not to take $f_{\pi}$ from experiment but rather to adjust $f_{\pi}$ and $g$ to give the nucleon mass and the nucleon-delta splitting correctly. We take $f_{\pi}=6.45$ $\mathrm{MeV}, g=4.48$ and use $m_{\pi}=138 \mathrm{MeV}$. It should be kept in mind that all the results on the static $N N$ force and on the spin-orbit interaction are based on only these three parameters. As was discovered by Skyrme the model has a topologically conserved quantum number, which is identified as the baryon number, $B$. The $U$ field is interpreted as a combination of a scalar $\sigma$ field and an iso-vector pion field, $U=(\sigma+i \vec{\tau} \cdot \vec{\pi}) / f_{\pi}$. The $\sigma$ field is not an independent physical field due to the unitarity constraint on $U$.

The standard time-independent solution to the classical field equations for $B=1$ is the defensive hedgehog, where the pion field points radially outward,

$$
U_{1}(\vec{r})=\exp (i \vec{\tau} \cdot \hat{r} f(r))
$$

The baryon number of this state is given by $B=(f(0)-f(\infty)) / \pi=1$. This solution breaks translational invariance, as well as the $O(4)$ spin-iso-spin symmetry, but the sum of spin and iso-spin is still conserved. If we perform a global $\mathrm{SU}(2)$ iso-rotation on the state,

$$
U_{1}(\vec{r} \mid A)=A^{\dagger} U_{1}(\vec{r}) A
$$


we obtain a state of the same energy.

For the $B=2$ system, we will use the product Ansatz. This Ansatz makes use of the fact that the product of two $B=1$ solutions has baryon number two. The most general Ansatz we can construct from two hedgehogs consists of the product of two separated and rotated hedgehogs,

$$
\begin{aligned}
U_{2}(r \mid \vec{R} A B) & =A^{\dagger} U_{1}(\vec{r}-\vec{R} / 2) A B^{\dagger} U_{1}(\vec{r}+\vec{R} / 2) B \\
& =U_{2}(r \mid \vec{R} C D) \\
& =D^{\dagger} C^{1 / 2 \dagger} U_{1}(\vec{r}-\vec{R} / 2) C U_{1}(\vec{r}+\vec{R} / 2) C^{1 / 2 \dagger} D .
\end{aligned}
$$

In the last line of $(\mathbb{1})$ we have introduced the matrix $D$, that describes the rigid isorotation of the whole system, as well as a relative iso-rotation $C$. When $R$ is very large changing $C$ or $D$ does not change the energy of the solution. For smaller $R$, $D$ still generates a zero-mode (corresponding to broken iso-spin symmetry), but the energy will depend on $C$. The energy is also invariant under spatial rotation, due to the conservation of angular momentum $\vec{J}=\vec{L}+\vec{S}$.

In order to evaluate the kinetic energy, we make all the collective parameters $(\vec{R}, A, B)$ time dependent, and substitute the product Ansatz (4) into the Lagrangian. This leads to an effective Lagrangian containing a kinetic energy that is second order in the time derivatives due to the special nature of the Skyrme Lagrangian. Note that we have already used Galilean invariance to decouple the center-of-mass motion from the relative motion.

We have evaluated all terms in the kinetic energy. The terms can be expanded in tensors in $C$ and powers of $\hat{R}$ (as well as the velocities, of course). The tensors form a basis for the irreducible representations of the $O(4)$ formed from the four components of $C$. We find only symmetric representations of $O(4)$ appearing, $(\sigma, 0)$, with $\sigma$ even. Only $\sigma=0$ (the unit operator), $\sigma=2$ and $\sigma=4$ occur in the expansion. Each of these terms is multiplied by a coefficient function that depends only on the size of $R$. We find typically that these functions are numerically largest if $\sigma=0$, smaller if $\sigma=2$, and smallest if $\sigma=4$. Note that the $\sigma=4$ operators have zero matrix element in two nucleon states. We also find several non-zero coefficients that are not even under parity or the interchange of the two Skyrmions - a well known disease of the product Ansatz. In the remainder of the discussion we ignore these terms. The full expansion of the kinetic energy is quite complicated and will be reported on separately [8]. Here we concentrate on the spin-orbit coupling and only consider terms that are dominant in that expansion.

In order to quantize the kinetic energy, we must first invert the mass matrix to obtain the Hamiltonian [9]. In other words we perform the standard transformation from a Lagrangian,

$$
L=\frac{1}{2} \sum_{i j} \dot{q}_{i} M_{i j} \dot{q}_{j}-V(\vec{q}),
$$


to a Hamiltonian,

$$
H=\frac{1}{2} \sum_{i j} p_{i}\left(M^{-1}\right)_{i j} p_{j}+V(\vec{q}),
$$

with $p_{i}=\sum_{j} M_{i j} \dot{q}_{j}$ conjugate to $q_{i}$. We separate the mass matrix in terms of its dependence on the $O(4)$ label, $\sigma$,

$$
M=M_{0}+M_{2}+M_{4}
$$

As mentioned above these terms decrease rapidly in size with increasing $\sigma$. We take advantage of this fact to $\operatorname{drop} M_{4}$ and write

$$
M^{(-1)} \approx M_{0}^{-1}-M_{0}^{-1} M_{2} M_{0}^{-1}+\text { small terms }
$$

The $L S$ coupling already appears in $M_{0}$, as an off-diagonal terms coupling the velocity $\vec{v}=\dot{\vec{R}}$ to the sum of the two rotational velocities for the individual Skyrmions. If we denote the sum and difference of the rotational velocities by $\vec{\omega}^{ \pm}$, we find that the dominant contribution of $M_{0}$ to the kinetic energy is given by

$$
K=\frac{1}{2}\left\{2 d_{1}^{v}(R) v^{2}+d_{1}^{\omega}(R)\left(\omega^{+^{2}}+\omega^{-2}\right)+2 u_{3}^{v \omega}(R)\left(\vec{v} \times \vec{R} \cdot \vec{\omega}^{+}\right)\right\} .
$$

Here the quantities $d_{1}^{v}, d_{1}^{\omega}$, and $u_{3}^{v \omega}$ are functions of $R$, calculated from the Skyrmion configurations. We have that $d_{1}^{v} \stackrel{R \rightarrow \infty}{\longrightarrow} M / 4, d_{1}^{\omega} \stackrel{R \rightarrow \infty}{\longrightarrow} 2 \Lambda$ and $u_{3}^{v \omega} \stackrel{R \rightarrow \infty}{\longrightarrow} 0$ ( $M$ is the mass of a single Skyrmion, and $\Lambda$ its moment of inertia.)

The approach in 过, 四 was to replace $\vec{v}$ by $\vec{p} /(M / 2)$, and $\vec{\omega}^{+}$by $\vec{S} /(2 \Lambda)$ to find

$$
V_{L S}=\frac{u_{3}^{v \omega}(R)}{M \Lambda} \vec{L} \cdot \vec{S}
$$

However this procedure is not equivalent to inverting the mass matrix. The matrix to be inverted here is basically two-by-two with the spin-orbit coupling coming from the off diagonal part. As is well know the off diagonal terms in such a case change sign under inversion. If we carry out the inversion from (5) to (6) we indeed find for the spin orbit force

$$
V_{L S}=-\frac{u_{3}^{v \omega}}{2 d_{1}^{v} d_{1}^{\omega}-\left(u_{3}^{v \omega}\right)^{2}} \vec{L} \cdot \vec{S} \stackrel{R \rightarrow \infty}{\longrightarrow}-\frac{u_{3}^{v \omega}(R)}{M \Lambda} \vec{L} \cdot \vec{S} .
$$

Thus we find the correct sign for the spin-orbit interaction. In Fig. 1 we show our calculation for the iso-spin independent part of the spin-orbit interaction. We show two calculated curves compared with the BonnR potential [10]. One calculation uses the asymptotic values (large $R$ ) for $d_{1}^{v}$ and $d_{1}^{\omega}$ for all $R$ and neglects the $u_{3}^{v \omega}$ in the denominator (this is close to the spirit of [3]), while the other keeps all the terms in Eq. (11) with their full $R$ dependence. Both curves agree fairly well with phenomenology 


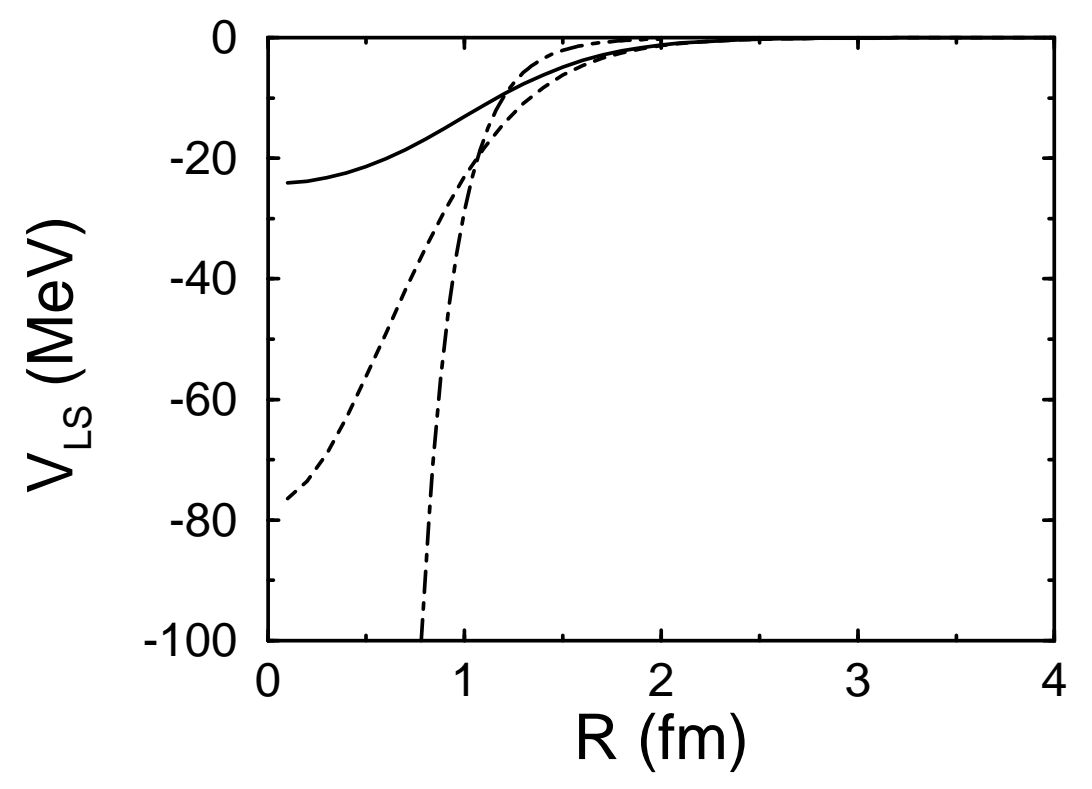

Figure 1: The isospin independent spin-orbit potential as a function of nucleon separation $R$. The solid line is calculated using the first expression in Eq. (11), whereas the dashed line represents the result from the second expression. The dash-dotted line is the corresponding term in the BonnR potential [10].

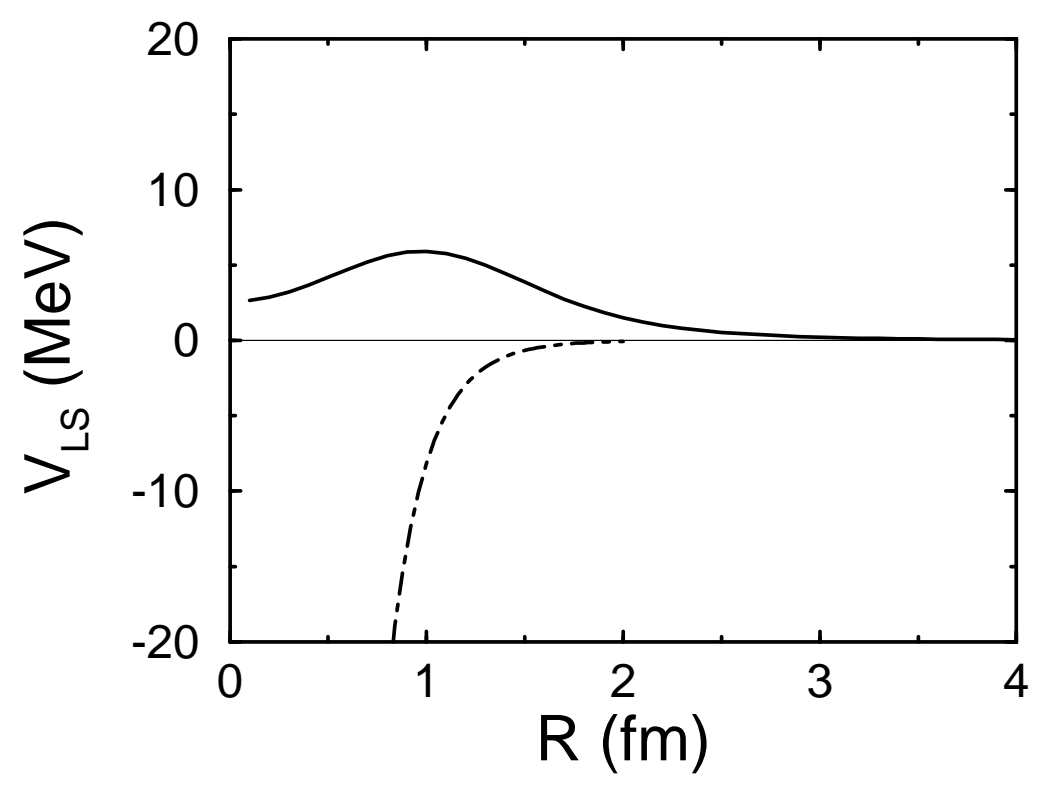

Figure 2: The isospin dependent spin-orbit potential as a function of nucleon separation $R$. The solid line is our result, and the dash-dotted line the corresponding term in the BonnR potential. 
beyond $1 \mathrm{fm}$, where the product ansatz makes sense, but differ at small distances. The $R$ dependent curve is probably the better indicator of what a full calculation will yield.

The iso-spin dependent part of the spin-orbit interaction comes from the $M_{2}$ term in Eq. (8). It is shown in Fig. 2 and compared with the BonnR potential [10]. As discussed above it is smaller than the iso-spin independent part, as is the data. It is also complicated with many terms contributing. As seen in the figure, it is positive at large $R$, and oscillates at smaller $R$. This oscillation comes from a cancellation among many terms and is probably quite sensitive to model assumptions. This sensitivity to cancellations recalls the static interaction in the central channel, suggesting that channel coupling may again be important [1].

Riska and Schwesinger [11] have added a 6th order term to the Skyrme model, and claim to find the right sign for the spin-orbit interaction. The results of this note shows that their discussion is faulty. It is not necessary to include a 6 th order term in the Skyrme model to find agreement between the model and phenomenology for spin-orbit coupling. Furthermore their 6th order term calculated as in (6) and (7), will lead to the wrong sign of the spin orbit interaction.

In summary we have shown that the Skyrme model yields the correct sign and order of magnitude for the $N N$ spin-orbit interaction. Coupled with our earlier demonstration that the Skyrme model correctly accounts for the main features of the static $N N$ interaction, one pion exchange, mid-range attraction, tensor force, short range repulsion, the result concludes successfully the first steps in the derivation of the $N N$ interaction from a starting point based in non-perturbative QCD.

This research was supported in part by the U.S. National Science Foundation.

\section{References}

[1] N. R. Walet et al, Phys. Rev. Lett. 68 (1992) 3849; N. R. Walet and R. D. Amado, Phys. Rev. C 47 (1993) 498; N. R. Walet, submitted to Phys. Rev. C.

[2] A. Jackson et al, Nucl. Phys. A432 (1985) 567; R. Vinh Mau et al, Phys. Lett. B150 (1985) 259; T. S. Walhout and J. Wambach, Phys. Rev. Lett. 67 (1991) 314.

[3] D.O. Riska and K. Dannbom, Phys. Scripta 37 (1988) 7.

[4] T.Otofuji et al, Phys. Lett. 205B (1988) 245.

[5] M. Oka, Phys. Lett. B175 (1986) 15; H. Odawara et al, Phys. Lett. B175 (1986) 115. 
[6] T. H. R. Skyrme, Proc. Roy. Soc. London 260 (1961) 127; 262 (1961) 237; Nucl. Phys. 31 (1962) 556.

[7] I. Zahed and G. E. Brown, Phys. Rep 142 (186) 1; K. F. Liu (ed.), Chiral Solitons, (World Scientific, Singapore, 1987), and references therein.

[8] Bin Shao, N. R. Walet and R. D. Amado, to be published.

[9] Goldstein, Classical Mechanics, (Addison-Wesley, Reading, 1980).

[10] C. Holinde et al, Phys. Rep. 149 (1987) 1.

[11] D.O. Riska and B. Schwesinger, Phys. Lett. 229B (1988) 339. 